\title{
Implementing Utility-Optimal CSMA
}

\author{
Jinsung Lee, Junhee Lee, Yung Yi, Song Chong*, Alexandre Proutière ${ }^{\dagger}$, Mung Chiang ${ }^{\ddagger}$ \\ ${ }^{*}$ Dept. of Electrical Engineering, KAIST, South Korea \\ Email: \{ljs,junhee\}@netsys.kaist.ac.kr, \{yiyung,song\}@ee.kaist.ac.kr \\ ${ }^{\dagger}$ Microsoft Research, Cambridge, UK \\ Email: alexandre.proutiere@microsoft.com \\ $\ddagger$ Dept. of Electrical Engineering, Princeton University, USA \\ Email: chiangm@princeton.edu
}

\begin{abstract}
Hundreds of papers over the last two decades have studied the theory of distributed scheduling in wireless networks, including a number of them on stability or utility maximizing random access. Several publications in 2008 studied an adaptive CSMA that in theory can approach utility optimality without any message passing under a number of assumptions. This paper reports the results from the first deployment of such random access algorithms through an implementation over conventional 802.11 hardware, an on-going effort that started in summer 2009. It shows both a confirmation that Utility Optimal CSMA may work well in practice even with an implementation over legacy equipment, and a wide array of gaps between theory and practice in the field of wireless scheduling. This paper therefore also brainstorms the discovery of and bridging over these gaps, and the implementation-inspired questions on modeling and analysis of scheduling algorithms.
\end{abstract}

Keywords: Scheduling, Random Access, CSMA, Optimization, Distributed Algorithm, Wireless Networks, Systems, 802.11.

\section{INTRODUCTION}

Design of distributed scheduling algorithms in wireless networks has been extensively studied under various metrics of efficiency and fairness and for different types of traffic and interference models. In their seminal work [1], Tassiulas and Ephremides developed a centralized scheduling algorithm, Max-Weight scheduling, achieving maximum stability, i.e., stabilizing any arrival for which there exists a stabilizing scheduler. Since then, there has been a large array of lowercomplexity, more distributed scheduling algorithms, using the ideas of randomization (pick-and-compare scheduling), weight approximation (maximal/greedy scheduling), or random access with queue-length exchanges, e.g., in [2]-[11], to achieve large stability region under unsaturated arrivals of traffic at each node. For saturated arrivals, optimizing a utility function, which captures efficiency and fairness at the equilibrium, has been studied for slotted-Aloha random access, e.g., in [12][17]. Together with the principle of Layering as Optimization Decomposition, advances in scheduling have also been translated into improvements in joint congestion control, routing, and scheduling over multihop wireless networks, e.g., [18][22]. There are many more studies on this topic, as discussed in more detail in surveys such as [23].

A main bottleneck that remains is the need for message passing in the above algorithms. Tradeoffs of the time complexity of message passing with throughput and delay have been studied recently, e.g., in [6], [7], [24] and [25]. Message passing reduces the "effective" performance, is vulnerable to security attacks, and makes the algorithms not fully distributed. This has lead to the following challenge on simplicity-driven design: Can random access without message passing approach some type of performance optimality? In theory, the answer was suggested to be positive in 2008, first in [26] for wireless networks, with a similar development in a different context in [27]. Convergence proof and tradeoff were presented in [28].

In [28], we developed a proof of the convergence of these algorithms without assuming that network dynamics freeze while the CSMA parameters are being updated, for the continuous-time Poisson clock model. New proof techniques were developed to overcome the difficulty of the coupling between the control of CSMA parameters and the queueing network dynamics. We then turned to more realistic slottedtime contention and backoff model, and quantified the effect of collisions. We revealed and characterized the tradeoff between long-term efficiency and short-term fairness: short-term fairness decreases significantly as efficiency loss is reduced. Similar to other distributed scheduling algorithms, there is a 3-dimensional tradeoff [24]: the price of optimality and zero message passing here is delay experienced by some nodes.

Given that a key goal in the above development of adaptive CSMA is to make high performance scheduling also simple: remove the need for message passing altogether, a natural question now becomes: if it is simple and near-optimal, it ought to be readily deployable to make a difference in some real network. This paper reports the results from the first deployment of such random access algorithms through an implementation over conventional 802.11 hardware, an ongoing effort that started very recently in summer 2009. It shows both a confirmation that Utility Optimal CSMA may work well in the practice even with an implementation over legacy equipment, and a wide array of gaps between theory and practice in the field of wireless scheduling. This paper therefore also brainstorms the discovery of and bridging over these gaps, and the implementation-inspired questions on modeling and analysis of scheduling algorithms. This is an "interim report" of the first set of outcomes and observations in the ongoing experiments, and further results will be presented in thefuture.

The rest of this paper is organized as follows: In Section II, 
we briefly describe the theoretical foundation of UO CSMA. Section III presents the implementation methodology and the analysis of the measurements collected from the experiments, followed by identifying the gaps between theory and practice in Section IV. Implementation-inspired theory and next steps of experimentation are outlined in Sections V and VI. We conclude the paper in Section VII.

\section{TheORY: Utility OPTIMAL CSMA}

In this section, we summarize UO CSMA (Utility-Optimal CSMA) that approximately achieves optimality in terms of total utility at equilibrium. We refer readers to [28] for details of the proofs for the results in Section II-E and II-F.

\section{A. Network and interference model}

We consider a wireless network composed by a set $\mathcal{L}$ of $L$ links. Interference is modeled by a symmetric, boolean matrix $A \in\{0,1\}^{L \times L}$, where $A_{k l}=1$ if link $k$ interferes with link $l$, and $A_{k l}=0$ otherwise. Denote by $\mathcal{N} \subset\{0,1\}^{L}$ the set of the $N$ feasible link activation profiles, or schedules. A schedule $m \in \mathcal{N}$ is a subset of non-interfering active links (i.e., for any $m \in \mathcal{N}, k, l \in m, A_{k l}=0$ ). We assume that the transmitters can transmit at a fixed unit rate when active. These assumptions on what constitutes "transmission" and "interference", together with several others later in the section, lead to mathematical tractability but also give rise to the theory-practice gaps that will be discussed in Section III and IV.

\section{B. Scheduling and utility maximization}

The network is assumed to handle single-hop data connections. However, the results presented here can be readily extended to multi-hop connections (e.g., using the classical back-pressure ideas [1]). The transmitter of each link is saturated, i.e., it always has packets to send. A scheduling algorithm decides at each time which links are activated. Denote by $\gamma^{s}=\left(\gamma_{l}^{s}, l \in \mathcal{L}\right)$ the long-term throughputs achieved by scheduling algorithm $s$. The throughput vector of any scheduling algorithm has to belong to the rate region $\Gamma$ defined by

$$
\begin{aligned}
\Gamma=\left\{\gamma \in \mathbb{R}_{+}^{L}: \exists \boldsymbol{\pi} \in \mathbb{R}_{+}^{N},\right. \\
\left.\forall l \in \mathcal{L}, \gamma_{l} \leq \sum_{m \in \mathcal{N}: m_{l}=1} \pi_{m}, \quad \sum_{m \in \mathcal{N}} \pi_{m}=1\right\} .
\end{aligned}
$$

In the above, for any schedule $m \in \mathcal{N}, \pi_{m}$ can be interpreted as the proportion of time schedule $m$ is activated. As is a standard in problems with saturated arrivals, the objective is to design a scheduling algorithm maximizing the total networkwide utility. Specifically, let $U: \mathbb{R}^{+} \rightarrow \mathbb{R}$ be an increasing, strictly concave, differentiable objective function. We wish to design an algorithm to solve the following optimization problem:

$$
\begin{array}{cl}
\max & \Sigma_{l \in \mathcal{L}} U\left(\gamma_{l}\right), \\
\text { s.t. } & \gamma \in \Gamma .
\end{array}
$$

We denote by $\gamma^{\star}=\left(\gamma_{l}^{\star}, l \in \mathcal{L}\right)$ the optimizer of (1). Most distributed schemes proposed in the literature to solve (1) make use of a dual decomposition of the problem into a rate control and a scheduling problem: A virtual queue is associated with each link; a rate control algorithm defines the rate at which packets are sent to the virtual queues, and a scheduling algorithm decides, depending on the level of the virtual queues, which schedule to use with the aim of stabilizing all virtual queues. The main challenge reduces to developing a distributed and efficient scheduling algorithm. Many solutions proposed so far are semi-distributed and require information about the queues to be passed around among the nodes or links. This signaling overhead increases communication complexity and reduces effective throughput. More importantly, for management and security reasons, practical deployment of scheduling algorithms is unlikely going to allow such message passing.

\section{Efficiency of CSMA}

CSMA-based random access is the most popularly used distributed scheduling algorithms in wireless networks and does not require any message passing. They are based on random back-off algorithms such as the Decentralized Coordinated Function (DCF) in IEEE802.11.

The network dynamics under CSMA have been extensively studied in the literature. The following popular model is due to Kelly [29], and has been recently revisited by e.g. [30] and [31]. In this model, the transmitter of link $l$ waits an exponentially distributed random period of time with mean $1 / \lambda_{l}$ before transmitting, and when it initiates a transmission, it keeps the channel for an exponentially distributed period of time with mean $\mu_{l}$. This CSMA algorithm is denoted by $\operatorname{CSMA}\left(\lambda_{l}, \mu_{l}\right)$ in the rest of the paper. Define $\boldsymbol{\lambda}=\left(\lambda_{l}, l \in \mathcal{L}\right)$ and $\boldsymbol{\mu}=\left(\mu_{l}, l \in \mathcal{L}\right)$. When each link $l$ runs $\operatorname{CSMA}\left(\lambda_{l}, \mu_{l}\right)$, the network dynamics can be captured through a reversible process [32]: If $m^{\boldsymbol{\lambda}, \boldsymbol{\mu}}(t)$ denotes the active schedule at time $t$, then $\left(m^{\boldsymbol{\lambda}, \boldsymbol{\mu}}(t), t \geq 0\right)$ is a continuous-time reversible Markov chain whose stationary distribution $\pi^{\boldsymbol{\lambda}, \boldsymbol{\mu}}$ is given by $\forall m \in \mathcal{N}, \quad \pi_{m}^{\boldsymbol{\lambda}, \boldsymbol{\mu}}=\frac{\prod_{l: m_{l}=1} \lambda_{l} \mu_{l}}{\sum_{n \in \mathcal{N}} \prod_{l: n_{l}=1} \lambda_{l} \mu_{l}}$, where by convention $\prod_{l \in \emptyset}(\cdot)=1$. Due to the reversibility of the process, the above stationary distribution does not depend on the distributions of the back-off durations or of the channel holding times, provided that they are of mean $1 / \lambda_{l}$ and $\mu_{l}$, respectively, for link $l$. This insensitivity property allows us to cover a more realistic scenario with uniformly distributed back-off delays and deterministic channel holding times.

Under the above continuous-time model, collisions are mathematically impossible, leading to tractability in this first step of the study. In practice, however, time is slotted and the back-off periods are multiple of slots, which inevitably causes collisions and will be discussed in Section II-F.

Under the $\operatorname{CSMA}\left(\lambda_{l}, \mu_{l}\right)$ 's algorithms, the link throughputs are given by

$$
\forall l \in \mathcal{L}, \quad \gamma_{l}^{\lambda, \mu}=\sum_{m \in \mathcal{N}: m_{l}=1} \pi_{m}^{\boldsymbol{\lambda}, \boldsymbol{\mu}}
$$

The following result proved in [26] (Propositions 1 and 2), states that any throughput vector $\gamma \in \Gamma$ can be approached using $\operatorname{CSMA}(\boldsymbol{\lambda}, \boldsymbol{\mu})$ algorithms. 
Lemma 1 ( [26]): For any $\gamma$ in the interior of $\Gamma$, there exist $\boldsymbol{\lambda}, \boldsymbol{\mu} \in \mathbb{R}_{+}^{L}$ such that $\forall l \in \mathcal{L}, \quad \gamma_{l} \leq \gamma_{l}^{\boldsymbol{\lambda}, \boldsymbol{\mu}}$.

The above lemma expresses the optimality of CSMA scheduling schemes, and it suggests that for approaching the solution of (1), one may use adaptive CSMA algorithms.

\section{Continuous time model: Algorithm and performance}

We now describe a generic adaptive CSMA-based algorithm to approximately solve (1). The algorithm is an extension of those proposed in [26], and does not require any message passing. Time is divided into frames of fixed durations, and the transmitters of each link update their CSMA parameters (i.e., $\lambda_{l}, \mu_{l}$ for link $l$ ) at the beginning of each frame. Each link maintains a virtual queue, denoted by $q_{l}[t]$ in frame $t$, for link $l$. The algorithm operates as follows:

\section{UO CSMA}

1) During frame $t$, the transmitter of link $l$ runs $\operatorname{CSMA}\left(\lambda_{l}[t], \mu_{l}[t]\right)$, and records the amount $S_{l}[t]$ of service received during this frame;

2) At the end of frame $t$, it updates its virtual queue and its CSMA parameters according to

$q_{l}[t+1]=\left[q_{l}[t]+\frac{b[t]}{W^{\prime}\left(q_{l}[t]\right)}\left(U^{\prime-1}\left(\frac{W\left(q_{l}[t]\right)}{V}\right)-S_{l}[t]\right)\right]_{q^{\min }}^{q^{\max }}$, and sets $\lambda_{l}[t+1]$ and $\mu_{l}[t+1]$ such that their product is equal to $\exp \left\{W\left(q_{l}[t+1]\right)\right\}$.

In the above algorithm, $b: \mathbb{N} \rightarrow \mathbb{R}$ is a step size function; $W: \mathbb{R}^{+} \rightarrow \mathbb{R}^{+}$is a strictly increasing and continuously differentiable function, termed the weight function; $V, q^{\min }, q^{\max }(>$ $\left.q^{\min }\right)$ are positive parameters, and $[\cdot]_{c}^{d} \equiv \min (d, \max (c, \cdot))$. We will later see that proper choice of $b$ ensures convergence. $V$ controls the accuracy of the algorithm, and the function $W$ controls the transient behavior. The impact of $b[t], V$, and $W$ will be demonstrated in the implementation in the next section.

Since the performance of CSMA algorithms depends on the products $\lambda_{l} \mu_{l}$ only, we have the choices in UO CSMA to either update the $\lambda_{l}$ 's (the transmission intensities) and fix the $\mu_{l}$ 's (the transmission durations), or to update the $\mu_{l}$ 's and fix the $\lambda_{l}$ 's, or to update both the $\lambda_{l}$ 's and $\mu_{l}$ 's.

\section{E. Convergence without timescale separation}

UO CSMA may be interpreted as a stochastic approximation algorithm with controlled Markov noise as defined in [33]. The main difficulty in analyzing the convergence of UO CSMA lies in the fact that the updates in the virtual queues, and hence in the CSMA parameters, depend on the random service processes $\left(S_{l}[t], t \geq 0\right)$. The service processes $\left(S_{l}[t], l \in \mathcal{L}\right)$ received by the various links in turn depend on the state of the network at the end of frame $t-1$, and on the updated CSMA parameters $(\boldsymbol{\lambda}[t], \boldsymbol{\mu}[t])$. We cannot assume that the network state "freezes" in between CSMA parameter updates. Without such a timescale separation assumption, proving convergence became more challenging [28].
For any vector $\boldsymbol{q} \in \mathbb{N}^{L}$, we denote by $\pi^{q}$ the distribution on $\mathcal{N}$ resulting from the dynamics of the $\operatorname{CSMA}(\boldsymbol{\lambda}, \boldsymbol{\mu})$ algorithms, where for all $l \in \mathcal{L}, \lambda_{l} \mu_{l}=\exp \left(W\left(q_{l}\right)\right)$. In other words,

$$
\forall m \in \mathcal{N}, \quad \pi_{m}^{q}=\frac{\exp \left(\sum_{l \in m} W\left(q_{l}\right)\right)}{\sum_{m^{\prime} \in \mathcal{N}} \exp \left(\sum_{l \in m^{\prime}} W\left(q_{l}\right)\right)} .
$$

We also denote by $\gamma[t]=\left(\gamma_{l}[t], l \in \mathcal{L}\right)$ the vector representing the cumulative average throughputs of the various links up to frame $t$, i.e.,

$$
\forall l \in \mathcal{L}, \quad \gamma_{l}[t]=\frac{1}{t} \sum_{n=0}^{t-1} S_{l}[n] .
$$

The next theorem states the convergence of UO CSMA under diminishing step-sizes, towards a point that is arbitrarily close to the utility-optimizer.

Theorem 1: Assume $\sum_{t=0}^{\infty} b[t]=\infty$ and $\sum_{t=0}^{\infty} b[t]^{2}<\infty$. For any initial condition $\boldsymbol{q}[0]$, UO CSMA converges in the following sense:

$$
\lim _{t \rightarrow \infty} \boldsymbol{q}[t]=\boldsymbol{q}_{\star} \text { and } \lim _{t \rightarrow \infty} \gamma[t]=\gamma_{\star} \text {, almost surely, }
$$

where $\gamma_{\star}$ and $\boldsymbol{q}_{\star}$ are such that $\left(\gamma_{\star}, \boldsymbol{\pi}^{q_{\star}}\right)$ is the solution of the following convex optimization problem (over $\gamma$ and $\boldsymbol{\pi}$ ):

$$
\begin{array}{ll}
\max & V \sum_{l \in \mathcal{L}} U\left(\gamma_{l}\right)-\Sigma_{m \in \mathcal{N}} \pi_{m} \log \pi_{m} \\
\text { s.t. } & \gamma_{l} \leq \sum_{m \in \mathcal{N}: m_{l}=1} \pi_{m}, \sum_{m \in \mathcal{N}} \pi_{m}=1 .
\end{array}
$$

Furthermore UO CSMA approximately solves (1) as

$$
\left|\sum_{l \in \mathcal{L}}\left(U\left(\gamma_{\star, l}\right)-U\left(\gamma_{l}^{\star}\right)\right)\right| \leq \log |\mathcal{N}| / V .
$$

\section{F. Slotted time model: Performance and tradeoff}

In any real systems, time is slotted and collisions may occur. In this section, we briefly summarize the impact of collisions (see [28] more details). We consider the following model for slotted CSMA: The transmitter of link $l$ starts a transmission at the end of a slot with probability $p_{l}$ if the slot has been sensed to be idle. When a link is active, it can experience either a successful transmission or a collision. When a link is currently successfully transmitting, it releases the channel with probability $1 / \mu_{l}$ at the end of a slot. Collisions are classified into two kinds:

(a) Short collisions. Using a channel probing mechanism using a small signaling message, e.g., RTS/CTS, we restrict the length of collisions to a short time interval.

(b) Long collisions. Long collisions occur when RTS/CTSlike procedures are not implemented, so that collision time last for a maximum of holding times of links involved with collision.

If we want the resulting link throughputs of UO CSMA to be close to the solution of (1), the products of the transmission probabilities and the channel holding times need to be very large. In the adaptation of UO CSMA to the slotted-time scenario, this implies that the channel holding times are very large, since the transmission probabilities must remain very 
small (to ensure very low collision rates). This further implies that the delay between two successive successful transmissions on a link is very large as well. In other words, to ensure efficiency, we need to sacrifice short-term fairness.

Another source of short-term unfairness with UO CSMA is the fact that if a link is interfering with by a lot of links (compared to other links), before transmitting it needs to wait until all its neighbors become inactive. This waiting time can be very long, especially if these neighbors do not consistently sense each other. When the link finally gets access to the channel, it then needs to hold the channel for a duration that is much larger than the transmission durations of its neighbors, in order to achieve throughput fairness. This may considerably exacerbate short-term unfairness.

We summarize the quantification of the above two observations in [28]. We define the short-term fairness index of link $l$ as $1 / T_{l}$ where $T_{l}$ is the average delay between two successive successful transmissions on this link. To illustrate, consider a simple star network: it is composed of $L+1$ links, where link 1 interferes with by all other links, but link $k, k>1$, interferes with only link 1 . Throughout some computations (see [28] for details), it turns out that for a given efficiency loss $\epsilon>0$, (i) channel holding times for link 1 and link $k, k>1$ scale as $1 / \epsilon^{2 L}$ and $1 / \epsilon^{2}$, respectively, and (ii) the short-term fairness for all links scales as $\epsilon^{2 L}$. This quantifies the tradeoff between efficiency and short-term fairness when implementing UO CSMA in slotted-time systems, implying that a substantial cost of short-term unfairness needs to be paid for large efficiency.

\section{Practice: Implementation of UO CSMA}

This section describes the implementation of UO CSMA on the 802.11-based conventional hardware platform and presents the first set of preliminary results. This implementation provides a proof-of-concept of the theory-driven algorithm, UO CSMA, and addresses the key challenges to transfer from theory to practice in distributed scheduling. In Section IV, we elaborate on the gaps between theory and practice and the workaround solutions to bridge some of them.

\section{A. WiMesh Network and Common Code Architecture}

We implemented UO CSMA on a campus-scale mesh network at KAIST, Korea, the WiMesh testbed [34]. The WiMesh has 56 mesh routers in an office building and in 6 undergraduate dormitory buildings over $1 \mathrm{~km}^{2}$ area, as shown in Figure 1. Each node is typically equipped with two $802.11 \mathrm{a} / \mathrm{b} / \mathrm{g}$ based wireless interface cards as well as one Ethernet interface. The WiMesh has been designed for a research testbed that is open to researchers who want to test and realize their conceptual ideas on top of real hardwares.

One of the unique features in WiMesh is Common Code Architecture (CCA), a programming environment designed to reduce cost and effort of testing a protocol by providing a way of using one code for both simulation and physical experiment. A typical way to validate new algorithms or protocols is to first make simulation programs at e.g., [35],

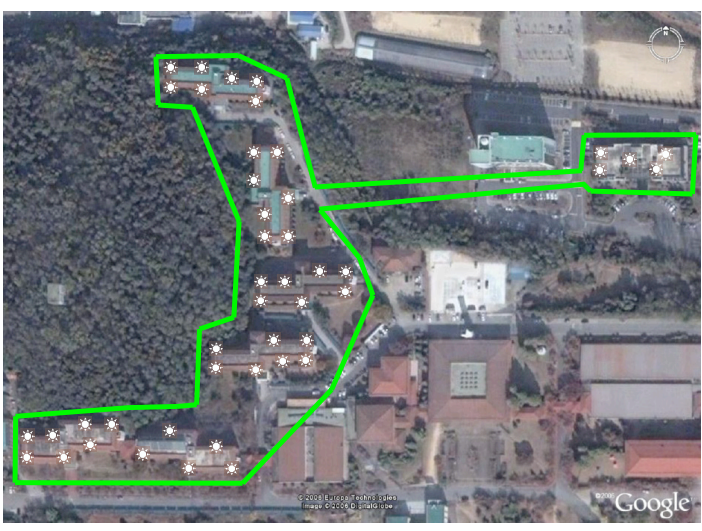

Fig. 1. WiMesh: Campus-wide wireless mesh network testbed at KAIST.

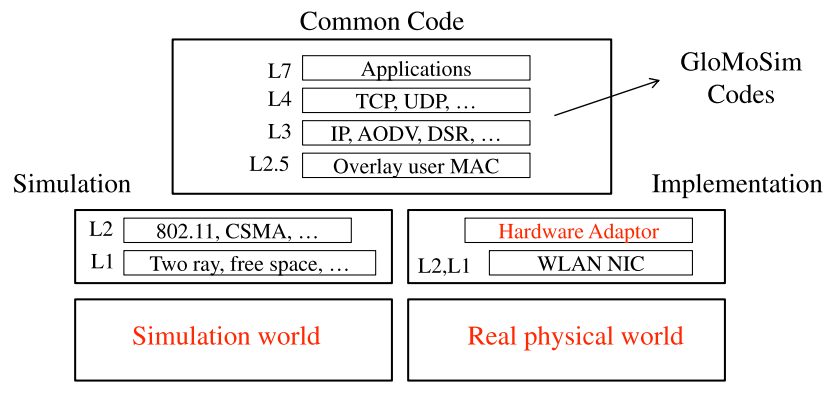

Fig. 2. Common Code Architecture: GloMoSim simulation codes are reusable in WiMesh without modification.

[36] and then implement them in real hardware. However, implementing in a physical network is significantly different from simulations and much more challenging, as it requires in-depth understanding of network protocol stacks, skills of system programming, and even modification of proprietary hardware through work-around hacking. Deploying protocols designed from a substantially new angle, such as UO CSMA, is particularly hard compared to simple modifications of existing protocols, e.g., 802.11 DCF with a new backoff scheme. CCA reduces the transition time from theory to simulation and then to implementation.

In CCA, we first make a simulation code using the protocol stack of GloMoSim simulator [36]. Once simulation tests verify the developed protocol, we load and run the same simulation codes on top of CCA. Most of functions of CCA are run at user-level space which is possible by making interfaces connecting between user-level processes and wireless hardwares. Figure 2 depicts the structure of CCA. We refer readers to [37] for more details.

\section{B. Setups of Simulation and of Implementation}

To evaluate performance, we compare UO CSMA with the optimal benchmark and the standard 802.11 DCF in both simulation and implementation. In simulation, we implement UO CSMA by changing the CSMA in GloMoSim, where we mainly modified the part that sets backoff counters. We used two-ray path-loss model, SNR bounded packet reception. We disabled ACK operation, so when collision occurs, it lasts for the corresponding holding time. The network is slotted with 
1.6ms timeslot and 5Mbps link capacity, and the packet size is set to be 1000 bytes.

Through CCA, we then use the same GloMoSim code to experiment UO CSMA in real hardware after a series of hacking that induces the underlying 802.11 drivers to effectively execute the UO CSMA algorithm. The setup and hardware specification is shown in Table I.

TABLE I

ENVIRONMENTAL SETUP FOR EXPERIMENT

\begin{tabular}{|c|c|}
\hline WLAN device & Atheros 5212 chipset \\
\hline PHY & $802.11 \mathrm{a}, 5.745 \mathrm{GHz}$ band, 6Mbs rate \\
\hline Flow & Single-hop session \\
\hline Traffic & Saturated \\
\hline Utility function & $U(x)=\log (x)$ \\
\hline Performance metrics & Total utility (or, throughput), short-term fairness \\
\hline Holding time & $20,100,500($ packets) \\
\hline V parameter & $20,100,500$ \\
\hline Weight function & $W(x)=x$ and $\log \log (x)$ \\
\hline
\end{tabular}

We summarize an example of the hacking needed to overlay UO CSMA algorithm over 802.11 hardware. In 802.11, contention resolution scheme operates based on the contention window, $C W$, where a back-off counter randomly chosen in the range $[0, C W-1]$. We modify the mechanism of setting $C W$ appropriately so that 802.11 drivers can be turned into a basis for implementing UO CSMA.

(a) Per-link $C W$. In $802.11, C W$ is maintained at each node, not each link, i.e., one contention window per one interface card. In UO CSMA, backoff counters should be installed per link. We implemented per-link $C W$, denoted by $C W_{l}$, at GloMoSim, and associate per-link $C W_{l}$ to the per-node $C W$, whenever link $l$ is activated.

(b) From access probability to contention window. Our theory is developed based on access probability $p_{l}$. Thus, we need a way of converting $p_{l}$ to $C W_{l}: C W_{l}=2 / p_{l}$, where ' 2 ' is needed since the actual contention window size is selected randomly from $\left[0, C W_{l}-1\right]$.

(c) $C W_{\min }$ and $C W_{\max }$ values. In 802.11, there exist two back-off related values. The real $C W$ is first set to be $C W_{\min }$ and then doubles whenever there is a collision. The doubled $C W$ value is used when a collided packet is retransmitted. We disable this feature by setting $C W_{\min }=$ $C W_{\max }$, so that retransmitted packets are not treated in a special way.

Note that in our physical experiments, both UO CSMA and 802.11 DCF are implemented through CCA, implying there is no gap due to Common Code between them. To facilitate packet-by-packet parameter control with less overhead, instead of using (indirect) interfaces such as user-level commands, e.g., iwpriv, provided by the device driver, we directly instill the target parameter into the so-called TX descriptor used by the firmware to make decision.

\section{Results of 3-link Experiment}

Now we present the preliminary results in a simple 3 link topology, as shown in Figure 3, where flows 1 and 2 (2 and

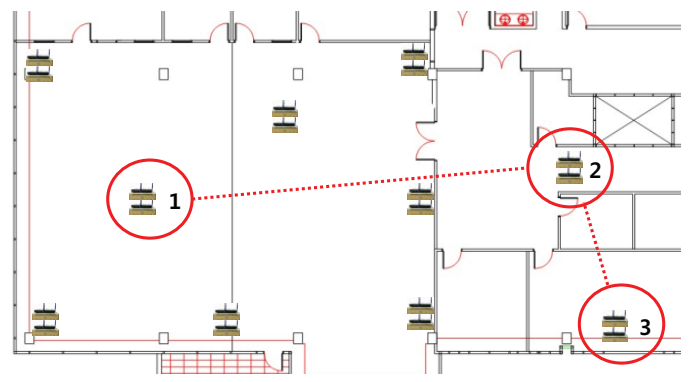

(a) A network topology with three links and six nodes in experiment on WiMesh testbed.

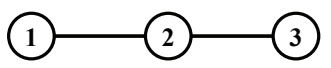

(b) Interference graph: We map a link to a node, where two nodes are if they are interfering with each other.

Fig. 3. Network topology in our experiment.

3 , respectively) are interfering with each other, but flows 1 and 3 do not interfere. In physical reality, interference among links cannot be modeled by a simple graph, since channels are time-varying and interference is very often asymmetric. We tried various placements of wireless nodes in WiMesh network, so that we obtain the desired interference relationship in Figure 3. While numerical results from any one topology cannot be extrapolated by too much, this small experiment already allows us to get a deep view on many aspects of theory prediction, including performance, transient behavior, and parameter setting. Ongoing work will extend these to large topologies.

\section{Total utility and throughput deviation}

We first performed multiple per-link throughput measurement without any interference to figure out effective link capacity without MAC overhead. In our hardware setup, the average per-link capacity amounts to about $5 \mathrm{Mbps}$. We henceforth use this value as a link capacity.

In theory, it is easy to compute the optimal throughput by solving (1) with $\log$ utility function for the proportionally fair solution, where $\gamma_{2}^{*}=1 / 3 \times 5 \approx 1.67 \mathrm{Mbps}$, and $\gamma_{1}^{*}=\gamma_{3}^{*}=$ $2 / 3 \times 5 \approx 3.33$ Mbps. In simulation, we implemented UO CSMA with adaptive backoff scheme on top of fixed parameter CSMA. The simulation almost matches the model over the slotted model in Section II, except that access probability is replaced by contention windows. Implementation over 802.11 hardware then introduces many more differences.

Figure 4 summarizes the results, averaged over many instances of experiments, where we show the total utilities over links as well as deviation of achieved throughputs from the optimal values. The deviation percentage is computed by normalizing the total throughput difference from optimal one for each case.

Figure 4 shows that UO CSMA in simulation has a very close match with the continuous-time-model-based theory, where the small discrepancy is mainly due to the difference 


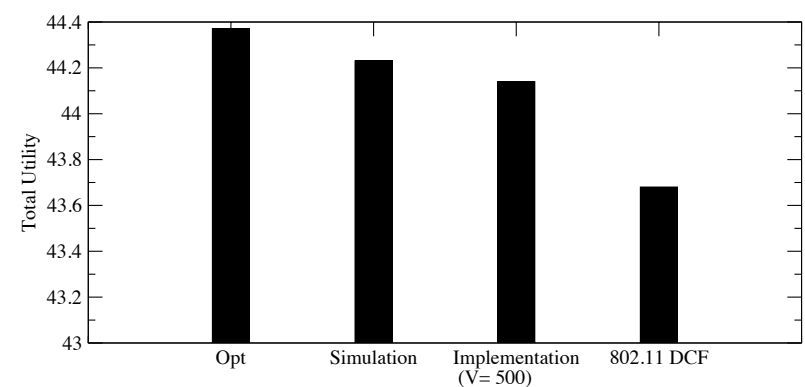

(a) Total utility for theory, simulation and implementation of UO CSMA, and 802.11 DCF.

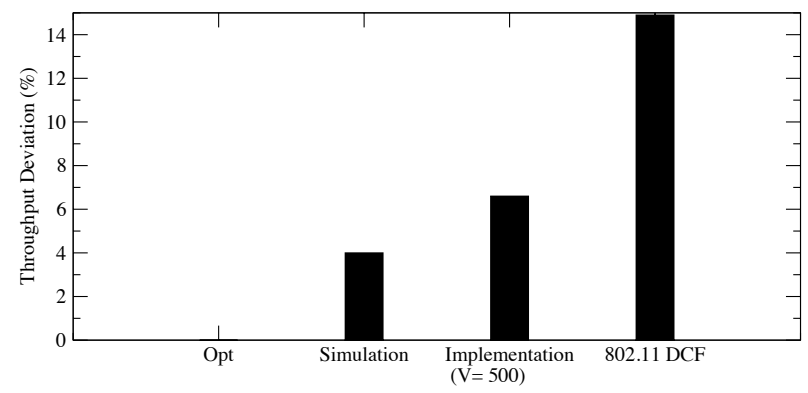

(b) Throughput deviations from theory.

Fig. 4. Total utility and throughput deviation.

between the ideal continuous time model without collision and the slotted time model with collision. More importantly, we also observe that UO CSMA implementation over 802.11 works well, with $6.6 \%$ throughput deviation from theory, whereas about $14.9 \%$ throughput deviation is observed in 802.11 DCF. UO CSMA in implementation recovers about $80 \%$ of the difference between DCF and simulated UO CSMA. This presents the first hardware-based confirmation that UO CSMA may work well despite the many mismatches between its assumptions and the physical reality of the network and devices.

\section{Holding time: Efficiency and short-term fairness}

Section II-F shows that increasing holding times lead to an increase in total throughput, at the expense of shortterm fairness. Recall that we define short-term fairness as the inverse of longest starvation time, where starvation time is the interval between two successful transmission. Poor short-term fairness may also lead to degradation in TCP performance. We measured such tradeoff between throughput and short-term fairness and show it in Figure 5.

From Figure 5, we observe that, as holding time increases, the short-term fairness decreases, just as theory predicted, whereas throughput remains about the same, contrary to the expectation based on theory. To figure out why, we performed the following measurements: By modifying the Madwifi device driver [38], we disabled retransmission. We assume that there is negligible packet loss due to channel attenuation, since each sender-receiver pair has a small transmission distanced. For different holding times, we observe a similar number of collisions around $0.05 \%$, There was little dependency of the number of collisions on holding time. We conjecture that (i) there are effective contention avoidance mechanisms, at least

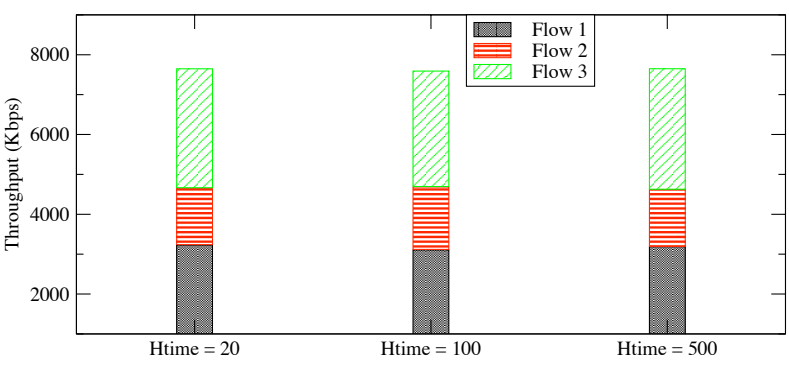

(a) Throughput for different holding times (in packets).

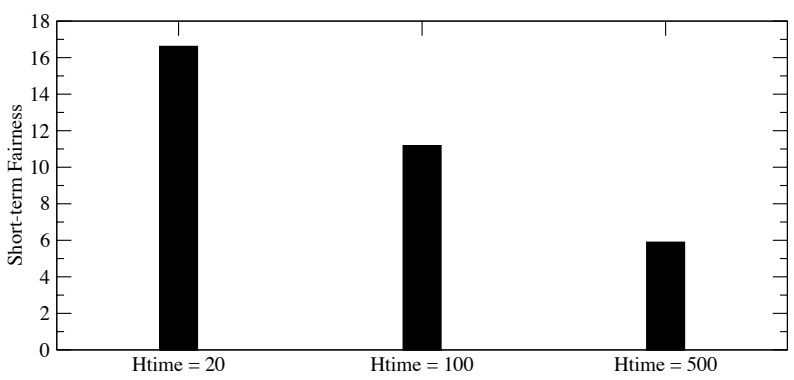

(b) Short-term fairness for different holding times (in packets).

Fig. 5. Throughput and short-term fairness.

for small networks, such as the arbitration by backoff in 802.11 DCF, in spite of small CW values (for small holding times), and (ii) asynchronous operation of 802.11 induced by delay such as the turnover time between transmission and reception and transition time for carrier sensing. More measurements are necessary to verify our conjectures.

\section{Step size: Convergence and transient behavior}

We also observe the impact on convergence and the queue behavior by different step sizes. As shown in Figures 6, the trajectories with the step size $b=0.01$ are oscillating within some neighborhood of the converged point: 0.4 for flow 2 and 0.2 for flow 3 . The trajectories with decreasing step size (reduced by 0.9 every 10 seconds starting from 0.01 ) converges within a few hundred seconds. This convergence is in terms of queue length, while the convergence in terms of throughput is already achieved in both cases. This confirms the standard result from optimization theory in this case: diminishing stepsize helps ensure convergence. It is more interesting, however, to note that the average throughput achieved with (a small) constant stepsize is almost identical to that achieved with diminishing stepsize. Therefore, if queue fluctuation is not an issue (e.g., for upper layer protocols like TCP), constant step size works just fine on throughput and the associated utility.

\section{Parameter V: Utility optimality and average delay}

According to theory, parameter $V$ in UO CSMA controls how accurately it solves the utility optimization problem: bigger $V$ gets closer to utility optimality. It also controls the sensitivity of response to network congestion that is reflected in the virtual queue lengths $\boldsymbol{q}[t]$. Bigger $V$ results in higher throughput, yet larger virtual queue lengths (thus longer delay). Figures 7 and 8 show throughput changes and virtual queue behaviors for $V=20,100$, and 500, respectively. For the small $V=20$, we observe that the virtual queue length 


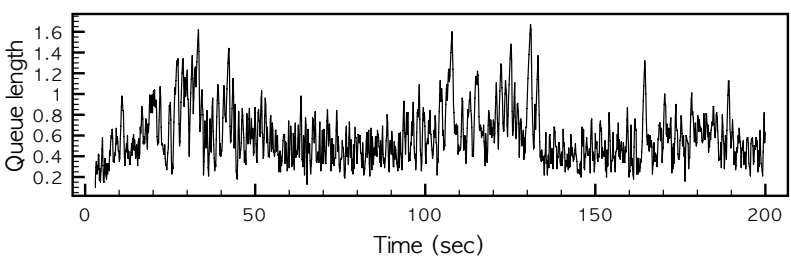

(a) Queue trace of flow 2 with fixed step size.

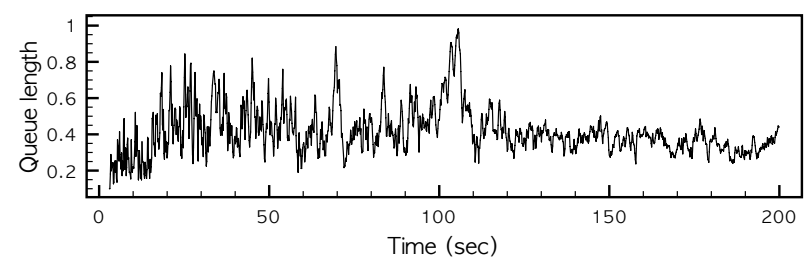

(b) Queue trace of flow 2 with diminishing step size.

Fig. 6. Flow 2 converges to 0.4 in both cases.

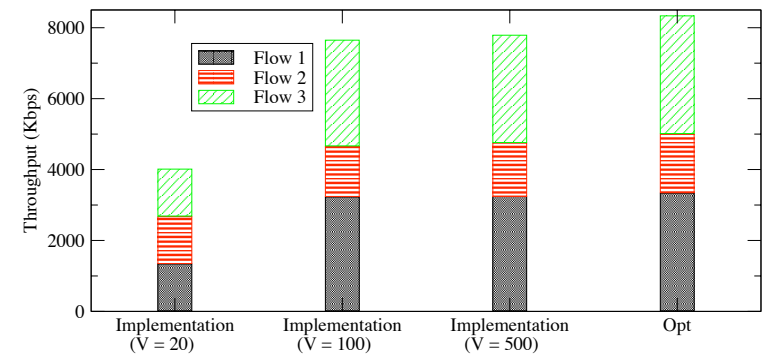

Fig. 7. Throughput for $\mathrm{V}=20,100,500$ and optimality.

constantly stays at the pre-specified minimum value (i.e., 0.1). Conversely, for the large $V=500$, the virtual queue length reaches the pre-specified maximum (i.e., 2.3), and stays there from 22 secs on. Note that the virtual queue is nothing but a real queue length multiplied by step size when $\log$ utility is used, i.e., $\boldsymbol{q}[t]=\boldsymbol{Q}[t] \times \boldsymbol{b}[t]$, where $\boldsymbol{Q}[t]$ means the actual queue length. Typical lengths of our actual queue range from 100 to 300 packets.

As a remark, our system is saturated and consists of singlehop sessions, where the virtual queue is used as a control variable to derive the system towards different directions. However, in the implementation of UO CSMA, we install perlink intermediate buffers to whom packets are injected (from an infinite backlogged reservoir) at a congestion controlled rate. The average length of such intermediate buffers indirectly measures the average queueing delay that a packet experiences.

\section{Weight function $W$ : Queue length and convergence time}

We next investigate the transient behaviors of queue length and throughput for different weight functions. We tested two popular functions: $W(x)=x$ and $W(x)=\log \log (x+e)$. As depicted in Figures 9 and 10, we can observe that $W(x)=$ $x$ has smaller virtual queue length and longer convergence time than $W(x)=\log \log (x+e)$ with the same equilibrium throughput being achieved for both functions, just as theory predicted. The virtual queue lengths for $W(x)=x$ oscillate between 0.2 and 0.4 for $W(x)=x$, whereas between 0.5 and 1.5 for $W(x)=\log \log (x+e)$. This is due to the fact that the long-term throughput is determined by $\rho$ (thus $\rho$ should

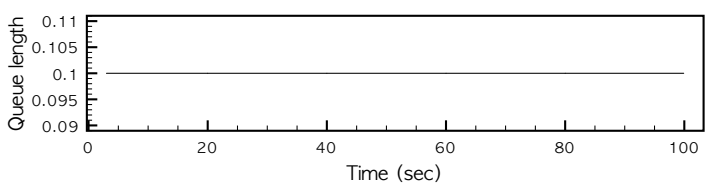

(a) Queue trace of flow 2 for $V=20$.

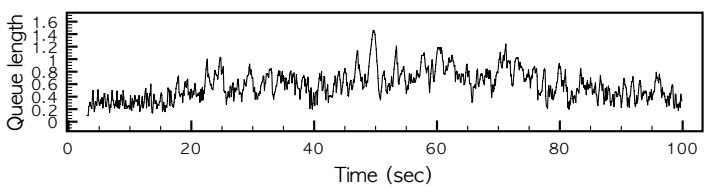

(b) Queue trace of flow 2 for $V=100$.

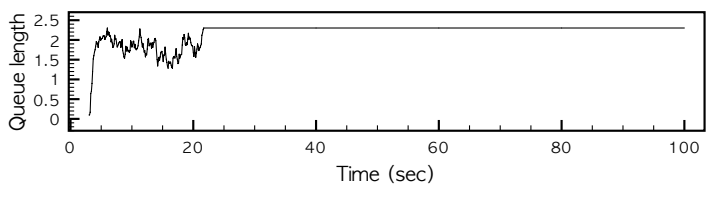

(c) Queue trace of flow 2 for $V=500$.

Fig. 8. Backlogs for different $\mathrm{V}$ parameters.

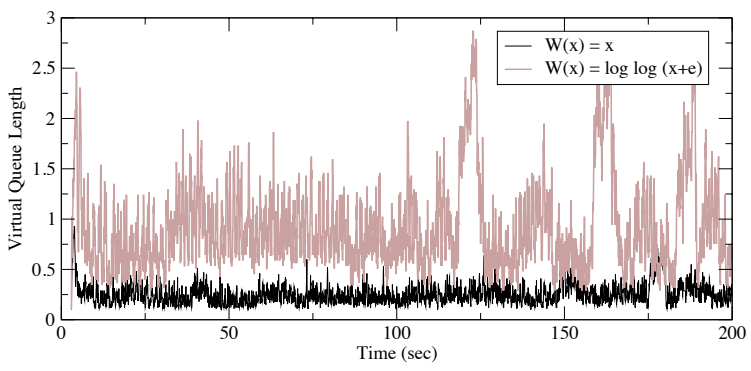

Fig. 9. Virtual queue traces for $W(x)=x$ and $W(x)=\log \log (x+e)$.

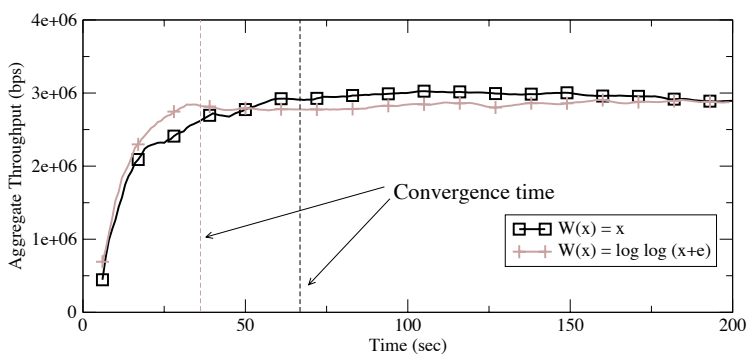

Fig. 10. Aggregate throughput traces and convergence times for $W(x)=x$ and $W(x)=\log \log (x+e)$.

be the same for both functions), but since $\rho=\exp (W(q))$, we have smaller virtual queue lengths for $W(x)=x$. On the other hand, we also observe faster convergence time for "less aggressive" weight function, i.e., $W(x)=\log \log (x+e)$. This may be due to the fact that less aggressive weight functions result in larger virtual queue length fluctuations, which, in turn, enables the protocol respond to congestion more sensitively. The proper choice of $W$ function is much less understood in theory than $V$ and $b[t]$ parameters, and these implementation results call for further study. 


\section{THEORY-PRACTICE GAPS}

\section{A. Overview of Gaps}

This section brainstorms the origins of many gaps between theory and practice based on our measurements. Such an understanding is important to improve theory-driven implementation of UO CSMA. In addition, it motivates us to develop better theories capturing and bridging this gap. The key gaps include the incomplete modeling of sensing, holding time, and interference in practice, as well as asymmetry of sensing and decoding ranges, asynchronism of device operations, and overhead within the protocol stacks. These will be discussed in three groups in the rest of this section.

Other gaps are caused by implementation methodologies. First, we adopt CCA to facilitate simulation and experiment, which, however, may lead to unexpected overhead as well as impact on the behaviors of networking, e.g., inappropriate event scheduling in CCA can starve transmission opportunity in the wireless interface. Second is imperfect controllability and visibility in an deployment over 802.11. We implement a new scheduling protocol on top of the conventional MAC hardware that are just partially controlled by us, with some features hidden from us (e.g., functions implemented at firmware). For example, when a contention window $C W$ is set by us, the system randomly chooses a backoff counter in the interval $[0, C W-1]$. We do not know the real backoff counter, which is sometimes needed to investigate the algorithm's behavior.

\section{B. Gap between Theory and Simulation}

We simulated UO CSMA under perfect synchronization over a slotted-time system by modifying the conventional CSMA with new CW-based backoff counter control mechanism. We also used a graph-based interference model. Carrier sensing is deterministic, and its range is set to be equal to transmission range. Consequently, as seen in Figure 11, there is a small gap between theory and simulation, which is mainly due to collisions in slotted-time model.

TABLE II

THEORY-SIMULATION GAP

\begin{tabular}{|c|c|c|}
\hline Gap & Theory & Simulation \\
\hline \hline Backoff & Data-slot based & Mini-slot based \\
\hline Collision & No & Yes, and last for holding time \\
\hline
\end{tabular}

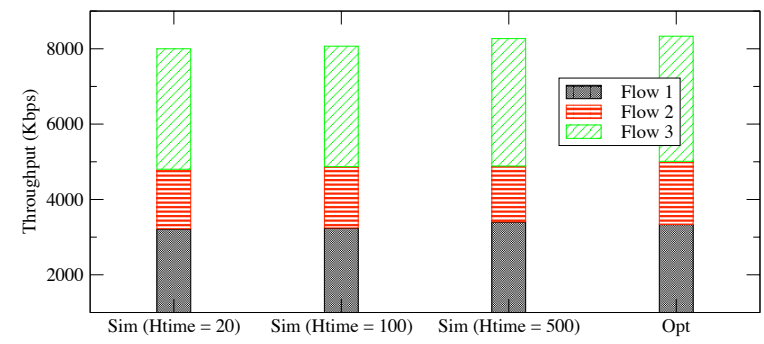

Fig. 11. Throughput in theory (Opt) and simulation (Sim) for holding time $=20,100$, and 500 packets.
TABLE III

SIMULATION-IMPLEMENTATION GAP

\begin{tabular}{|l|l|l|}
\hline Gap & Simulation & Implementation \\
\hline \hline Hidden-terminal & $\begin{array}{l}\text { No, due to link-based in- } \\
\text { terference model }\end{array}$ & $\begin{array}{l}\text { Yes, due to carrier sensing } \\
\text { by senders }\end{array}$ \\
\hline CS and TX ranges & Same & $\begin{array}{l}\text { Different and governed by } \\
\text { PHY layer }\end{array}$ \\
\hline $\begin{array}{l}\text { Time-varying } \\
\text { channel }\end{array}$ & No, fixed channel & $\begin{array}{l}\text { Yes, no guarantee of hold- } \\
\text { ing time }\end{array}$ \\
\hline Asymmetry & No, symmetric & $\begin{array}{l}\text { Yes, both at transmission } \\
\text { and carrier sensing }\end{array}$ \\
\hline
\end{tabular}

\section{Gap between Simulation and Implementation}

(1) Hidden-terminal: Link-oriented vs. node-oriented. Even if two nodes do not sense signal, their intended transmissions can collide. This is due to the gap between theory that interferences are characterized by links and practice that carrier-sensing is performed by nodes. The hiddenterminal problem can be a cause of throughput decrease in practice, when RTS/CTS mechanism can be a candidate solution. RTS/CTS-like signaling may also help with driving the system with slotted time from the continuoustime one in theory into one with short collisions (see Section II-F).

(2) Difference in sensing and decoding ranges. In $802.11 \mathrm{~b}$, systems are designed to be conservative, so that sensing range is larger than decoding range. Thus, transmissions may defer even if they can be successful in decoding. This difference in decoding and sensing ranges sometimes depends on the proprietary hardware features. For example, sensing mechanism of 802.11a in our deployment differs from $802.11 \mathrm{~b}$, in that nodes first try to decode a preamble of packets from neighbors (thus sensing range is equal to decoding range). When the preamble is not decodable due to some unpredictable behavior such as instantaneous hardware-malfunction, a sensing range that is smaller than the decoding range is applied for carrier-sensing. Furthermore, "collision" is defined in physical systems by received SIR rather than hop count, and sometimes exhibits "capture effect" for some decoders.

(3) Time-varying channel. Time-varying channels make it difficult to fully guarantee the desired holding times, which is crucial for both long-term efficiency and short-term fairness. The holding time set by a node $i$ cannot always be guaranteed by an interfering neighbor that decrements its backoff counter whenever signal is not sensed (due to factors such as time-varying channel), and leads to preemption of the transmission of node $i$. Time-varying channels also generate packet loss induced by channel degradation, which is not considered by the current theory.

(4) Asymmetry. Wireless links are often asymmetric because signals propagate differently between two nodes. Furthermore, link asymmetry is also time-varying. In contrast, in UO CSMA theory so far, both link channel conditions and interferences are symmetric and fixed. 


\section{Gap between Clean-slate and 802.11}

There have been many discussions on the vision of cleanslate design for the overall future of the Internet. As an alternative option for a "local surgery" such as UO CSMA, implementation over legacy hardware like conventional 802.11 also presents a least-resistance path from theoretical advance to practical impact. However, there are also challenges and gaps due to the reuse of legacy hardware. The three most prominent ones are summarized below.

\section{(1) Holding time}

Perfect execution of holding time relies on perfect carriersensing capability. This is clearly impossible over 802.11. We adopted several work-arounds to ensure that holding time is executed correctly as much as possible.

- We use AIFS (Arbitration Inter-Frame Space) from 802.11. The AIFS specifies an interval between packet transmissions. We set AIFS to be a large value only when a node first access the media right after its backoff counter reaches 0 , but to be a small value for back-toback transmissions in the middle of holding time. This heterogeneous setting of AIFS precludes a node from intercepting transmissions from other nodes being in the middle of holding time.

- In time-varying channels, even while a node $i$ is transmitting data, node $i$ 's interfering links sometimes decrement their backoff counters in case when interfering links may not sense node $i$ 's signal due to channel fluctuations. To prevent it, we use the NAV (Network Allocation Vector) option recording the amount of time during which neighbors should be silent irrespective of carrier-sensing. This solution is particularly useful when the packet with a NAV value is overheard and decoded by interfering neighbors.

\section{(2) CW parameter value's granularity}

The second gap is the coarse $\mathrm{CW}$ granularity that prevents us from controlling access intensity perfectly. The 802.11 allows only $2^{x}-1 \mathrm{CW}$ values for some integers $x$, which is again implemented in firmware in the chip used in our experiment. This makes it impossible to ensure $\mathrm{CW}=0$, which is needed in fully executing UO CSMA according to its theory.

\section{(3) 802.11-specific packets}

The third gap is due to packets receiving special treatment in 802.11, where examples include beacon packets (with high priority) that are used to identify neighbors. These beacon packets may interrupt normal data transmission, adversely affecting the holding times. To tackle this problem, we modified the device driver to minimize the impact of the packets for beacon signal by increasing beacon interval from $100 \mathrm{msec}$ to $5 \mathrm{sec}$, but further increase of beacon interval would lead to slow connectivity update.

\section{DEVElOPing IMPLEMENTATION-INSPIRED THEORY}

In a field like wireless scheduling that has become highly mathematical, implementation not only helps validate predic-
TABLE IV

Clean SLATE-OVER 802.11 GAP

\begin{tabular}{|l|l|l|}
\hline Gap & Clean slate & Over 802.11 \\
\hline \hline Holding & Perfect & Imperfect \\
\hline Contention control & By access prob. & $\begin{array}{l}\text { By discrete back- } \\
\text { off, but only } 2^{n}-1 \\
\text { CW value available }\end{array}$ \\
\hline Transmission type & User defined & Unicast with ACK \\
\hline Synchronization & $\begin{array}{l}\text { Synchronization } \\
\text { from PHY }\end{array}$ & Asynchronous \\
\hline Overhead & $\begin{array}{l}\text { Hardware } \\
\text { dependent }\end{array}$ & $\begin{array}{l}802.11 \text { chipset de- } \\
\text { pendent }\end{array}$ \\
\hline
\end{tabular}

tions from theory and falsify assumptions behind theory, it also clarifies the paths for future study on more realistic and less tractable models.

From the initial results reported here, it is clear that the following topics deserve a closer look from the theory community:

- The impact of what existing theory assumed away, e.g., overhead, asymmetry of sensing and decoding, granularity of control parameters.

- The impact of what existing theory modeled simplistically, e.g., imperfect holding and sensing that are noised versions of the accurate operation.

- Sharper understanding of what theory has analyzed only loosely so far, e.g., convergence speed, transient behavior like queue buildup, and choices of $b[t], V$, and $W$ in striking the tradeoffs they each control.

\section{NeXt Steps in IMPLEMENTATION}

Only the first set of experimental results has been reported. Much remains to be done, including the following:

- Large-scale topology with multi-hop sessions. The next step is to test UO CSMA in part of WiMesh deployment consisting of more than 20 nodes with multi-hop sessions, where interactions with routing and transport protocols will introduce new challenges.

- Software and hardware upgrade. We plan to upgrade our testbed with $802.11 \mathrm{n}$ cards, as well as newer Linux kernels, with which more freedom to access the underlying hardware is provided.

\section{CONCLUDING REMARKS}

Theories depend on a mathematical crystallization of the engineering system under study. This process needs to ignore some parts of the physical characteristics of the system by making assumptions, simplify other parts by creating tractable models, and focus on metrics that can be crisply quantified and tightly analyzed using the mathematical machineries available. There is perhaps no other starting point towards a rigorous study. This paper aims at going one step further for the topic of distributed scheduling in wireless networks, through an implementation over conventional 802.11 hardware and a deployment in the WiMesh network at KAIST. This is simply an "interim report", where we report the first, small-scale experiment that confirms the ability of UO CSMA to get close to utility optimality despite many gaps between theory 
and practice. The discovery, quantification, and bridging of these gaps are more important than the numerical results. We identify the key gaps, group them in three types, and explain where they originated and how they might be bridged, either by work-around engineering solutions or by addressing the new modeling challenges through enriched theories of wireless scheduling in the future.

\section{ACKNOWLEDGEMENT}

We thank helpful discussions on UO CSMA with L. Jiang, J. K. Lee, J. Liu, H. V. Poor, R. Srikant, D. Shah, and J. Walrand. Part of this work has been supported in part by IT R\&D program of MKE/IITA [2009-F-045-01], Korea Research Council of Fundamental Science and Technology, and the Princeton EDGE Lab that is in part sponsored by the US NSF Computing Research Infrastructure program, the ONR Defense University Research Instrumentation Program, and Qualcomm.

\section{REFERENCES}

[1] L. Tassiulas and A. Ephremides, "Stability properties of constrained queueing systems and scheduling for maximum throughput in multihop radio networks," IEEE Transactions on Automatic Control, vol. 37, no. 12, pp. 1936-1949, 1992.

[2] L. Tassiulas, "Linear complexity algorithms for maximum throughput in radionetworks and input queued switches," in Proceedings of IEEE Infocom, San Francisco, CA, 1998.

[3] P. Chaporkar, K. Kar, and S. Sarkar, "Throughput guarantees through maximal scheduling in wireless networks," in Proceedings of the 43rd Annual Allerton Conference on Communication, Control and Computing, Monticello, IL, 2005.

[4] E. Modiano, D. Shah, and G. Zussman, "Maximizing throughput in wireless networks via gossiping," in Proceedings of ACM Sigmetrics, Saint Malo, France, 2006.

[5] A. Eryilmaz, A. Ozdaglar, and E. Modiano, "Polynomial complexity algorithms for full utilization of multi-hop wireless networks," in Proceedings of Infocom, Anchorage, AK, 2007.

[6] S. Sanghavi, L. Bui, and R. Srikant, "Distributed link scheduling with constant overhead," in Proceedings of ACM Sigmetrics, San Diego, CA, 2007.

[7] S. Ray and S. Sarkar, "Arbitrary throughput versus complexity tradeoffs in wireless networks using graph partitioning," in Proceedings of Information Theory and Applications Second Workshop, La Jolla, CA, 2007.

[8] C. Joo and N. B. Shroff, "Performance of random access scheduling schemes in multi-hop wireless networks," in Proceedings of Infocom, Anchorage, AK, 2007.

[9] Y. Yi and M. Chiang, "Wireless scheduling with $\mathrm{O}(1)$ complexity for m-hop interference model," in Proceedings of IEEE International Conference on Communications, Beijing, China, 2008.

[10] A. Gupta, X. Lin, and R. Srikant, "Low-complexity distributed scheduling algorithms for wireless networks," in Proceedings of IEEE Infocom, Anchorage, AK, 2007.

[11] X. Lin and S. Rasool, "Constant-time distributed scheduling policies for ad hoc wireless networks," in Proceedings of IEEE Conference on Decision and Control, San Diego, CA, 2006.

[12] K. Kar, S. Sarkar, and L. Tassiulas, "Achieving proportional fairness using local information in aloha networks," IEEE Transactions on Automatic Control, vol. 49, no. 10, pp. 1858-1862, 2004.

[13] J. W. Lee, M. Chiang, and R. A. Calderbank, "Utility-optimal medium access control: reverse and forward engineering," in Proceedings of IEEE Infocom, Barcelona, Spain, 2006.

[14] X. Wang and K. Kar, "Cross-layer rate optimization for proportional fairness in multihop wireless networks with random access," in Proceedings of ACM Mobihoc, Urbana-Champaign, IL, 2005.
[15] A. H. Mohsenian-Rad, J. Huang, M. Chiang, and V. W. S. Wong, "Utility-optimal random access: Optimal performance without frequent explicit message passing," IEEE Transactions on Wireless Communications, vol. 8, no. 2, pp. 898-911, 2009.

[16] - -, "Utility-optimal random access: Reduced complexity, fast convergence, and robust performance," IEEE Transactions on Wireless Communications, vol. 8, no. 2, pp. 898-911, 2009.

[17] J. Liu, A. Stolyar, M. Chiang, and H. V. Poor, "Queue backpressure random access in multihop wireless networks: Optimality and stability," IEEE Transactions on Information Theory, vol. 55, no. 9, 2008.

[18] X. Lin and N. B. Shroff, "The impact of imperfect scheduling on crosslayer rate control in wireless networks," in Proceedings of IEEE Infocom, Miami, FL, 2005.

[19] M. J. Neely, E. Modiano, and C. Li, "Fairness and optimal stochastic control for heterogeneous networks," in Proceedings of IEEE Infocom, Miami, FL, 2005.

[20] L. Chen, S. H. Low, M. Chiang, and J. C. Doyle, "Joint optimal congestion control, routing, and scheduling in wireless ad hoc networks," in Proceeding of IEEE Infocom, Barcelona, Spain, 2006.

[21] A. Eryilmaz and R. Srikant, "Joint congestion control, routing, and MAC for stability and fairness in wireless networks," IEEE Journal on Selected Areas of Communication (JSAC), Special Issue on Nonlinear Optimization of Communication Systems, vol. 24, no. 8, pp. 1514-1524, 2006.

[22] A. L. Stolyar, "Maximizing queueing network utility subject to statbility: greedy primal-dual algorithm," Queueing Systems Theory and Applications, vol. 50, no. 4, pp. 401-457, 2005.

[23] Y. Yi and M. Chiang, "Stochastic network utility maximization and wireless scheduling," 2009, to be published as a book chapter of NextGeneration Internet Architectures and Protocols, Cambridge University Press. Also, available at http://lanada.kaist.ac.kr/pubs/scheduling.pdf.

[24] Y. Yi, A. Proutiere, and M. Chiang, "Complexity in wireless scheduling: Impact and tradeoffs," in Proceedings of ACM Mobihoc, Hong Kong, China, 2008.

[25] Y. Yi, J. Zhang, and M. Chiang, "Delay and effective throughput of wireless scheduling in heavy traffic regimes: Vacation model for complexity," in Proceedings of ACM Mobihoc, New Orleans, LA, 2009.

[26] L. Jiang and J. Walrand, "A CSMA distributed algorithm for throughput and utility maximization in wireless networks," in Proceedings of the 46th Annual Conference on Communication, Control and Computing, Monticello, IL, 2008.

[27] S. Rajagopalan and D. Shah, "Distributed algorithm and reversible network," in Proceedings of the Cconference on Information Science and Systems, Princeton, NJ, 2008.

[28] J. Liu, Y. Yi, A. Proutiere, M. Chiang, and H. V. Poor, "Adaptive CSMA: Approaching optimality without message passing," Wiley Journal of Wireless Communications and Mobile Computing, Special Issue on Recent Advances in Wireless Communications and Networking,, Dec. 2009. Preliminary version in Microsoft Research Technical Report, TR2008-128, 2008.

[29] F. Kelly, "Stochastic models of computer communication systems," Journal of the Royal Statistical Society, vol. 47, no. 3, pp. 379-395, 1985.

[30] M. Durvy and P. Thiran, "Packing approach to compare slotted and non-slotted medium access control," in Proceedings of IEEE Infocom, Barcelona, Spain, 2006.

[31] C. Bordenave, D. McDonald, and A. Proutiere, "Performance of random medium access control: An asymptotic approach," in Proceedings of ACM Sigmetrics, Annapolis, MD, 2008.

[32] F. Kelly, Reversibility and Stochastic Networks. Wiley, Chichester, 1979.

[33] V. Borkar, "Stochastic approximation with controlled markov noise," Systems and control letters, vol. 55, pp. 139-145, 2006.

[34] S. Shrestha, J. Lee, A. Lee, K. Lee, J. Lee, and S. Chong, "An open wireless mesh testbed architecture with data collection and software distribution platform," in In Proceedings of TridentCom, Orlando, FL, 2007.

[35] "Ns-2," http://www.isi.edu/nsnam/ns/.

[36] "Glomosim," http://pcl.cs.ucla.edu/projects/glomosim/

[37] J. Lee, J. Lee, S. Shrestha, and S. Chong, "Common code architecture for future internet researches of wireless mesh networks," in Proceedings of CFI, Seoul, Korea, 2008.

[38] "Madwifi project," http://madwifi-project.org/. 